\title{
A New Acid Cleaning Method for Scale Removal in Condensers
}

\author{
Shuxia Xu, Ning Chen, Wei Peng \\ School of Electric Power Engineering, China University of Mining and Technology, Xuzhou 221116, \\ China
}

Keywords: Condenser; Pickling, Corrosion rate, Corrosion inhibitor, Stainless steel.

\begin{abstract}
Condenser scale should be paid more attention with the rapid development of society and industrial need. By hydrochloric immersion and washing with online cleaning robot, the descaling could be done efficiently. When the mass fraction of hydrochloric was $1.7 \%$, cleaning time will be less than three minutes, and the descale rate will be above $98 \%$. By electron microscopy analysis (SEM), it showed that corrosion spots appeared on the sample surface when stainless steel sheets were immersed in hydrochloric solution for 24 hours.
\end{abstract}

\section{Introduction}

The condenser is a cold source in the thermodynamic cycle of power plant. There is a major problem for it, which is the formation of scale in the inner wall of the condenser tubes at runtime. Previous research pointed out that scale formation is a combination of deposition and removal processes [1]. When the reclaimed water is heated up in the condensers, the solubility of the minerals decreases and precipitation occurs[2]. Reclaimed water forms variety of scale in the inner face of the condenser. In a recirculating system the buildup of non-volatile species within the cooling water is problematic because the increased concentration of non-volatile species leads to the precipitation of mineral salts [3]. Scale may cause one or more of several major operating problems:(1) loss of heat transfer, (2) under-deposit corrosion, (3) increased pressure loss, and (4) flow distribution[4]. Deposit removal is usually done either by a mechanic or chemical method. By comparison, a chemical cleaning method for condenser tubes is more appropriate, including online and offline cleaning. According to Harper W J [5], theoretical mechanism of chemical cleaning was proposed. Hydrochloric, nitric acid or amino sulfonic could be used to remove scale in chemical cleaning. The online cleaning process is easy to be carried out. It gets rid of temporary system and installation work, which reduces the pollution to environment. Therefore, online cleaning is a widely accepted method.

In this paper, an online and chemical cleaning method was applied. An underwater robot was used to clean condenser tubes. When adding certain concentration of hydrochloric to the robotic nozzles, the nozzles moved to one end of condenser tubes. Then hydrochloric was injected into the condenser tubes to immerse scale. Finally, circulating cooling water washed away the dirt out of the tubes, realizing the online cleaning.

\section{Principle}

\subsection{Chemical principle}

Descaling is conducted by the reaction between calcium carbonate and hydrochloric. The chemical reaction principle of the experiment were as followed.

$$
\begin{gathered}
\mathrm{Ca}\left(\mathrm{HCO}_{3}\right)_{2}=\mathrm{CaCO}_{3}+\mathrm{CO}_{2} \uparrow+\mathrm{H}_{2} \mathrm{O} \\
\mathrm{CaCO}_{3}+2 \mathrm{HCl}=\mathrm{CaCl}_{2}+\mathrm{H}_{2} \mathrm{O}+\mathrm{CO}_{2} \uparrow
\end{gathered}
$$

\subsection{Industrial requirements of pickling condenser}

During the operation of power plant, condenser tubes should be cleaned when the thickness of scale is bigger than $0.5 \mathrm{~mm}$. The experimental scale thickness was $0.5 \mathrm{~mm}$. The mass fraction of hydrochloric is usually about $3 \%$ as pickling condenser tubes in the power plants. In this paper, 
hydrochloric whose mass fraction was $1 \%-3 \%$ was used to immerse samples to study the smallest concentration. The corrosion rate should be smaller than $1 \mathrm{~g} /\left(\mathrm{m}^{2} \cdot \mathrm{h}\right)$ in the pickling process.

After condenser tubes pickling, the rest of chloride ion is harmful to condenser tubes of stainless steel, which will cause pitting corrosion, stress corrosion and so on because of its small atomic radius. The concentration of chloride ion shouldn't be higher than $700 \mathrm{ppm}$ of pickling solution. So, the concentration of chloride ion should be carried out validation.

\subsection{Work principle of online cleaning robot}

The potassium permanganate was used in the experiment. The online cleaning robot implemented cleaning by hydrochloric immersion. The cleaning system includes three parts: online cleaning robot, ultrasonic liquid level instrument (type of GL-100) and the robot online control system. The robot worked in the lower part of the condenser water chamber, and the power plant can continue to run when cleaning.

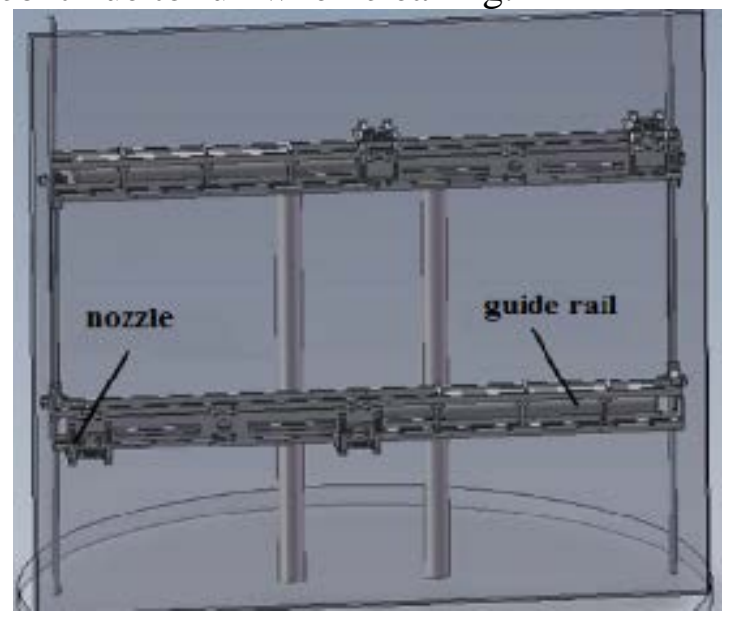

Figure1 The online cleaning robot

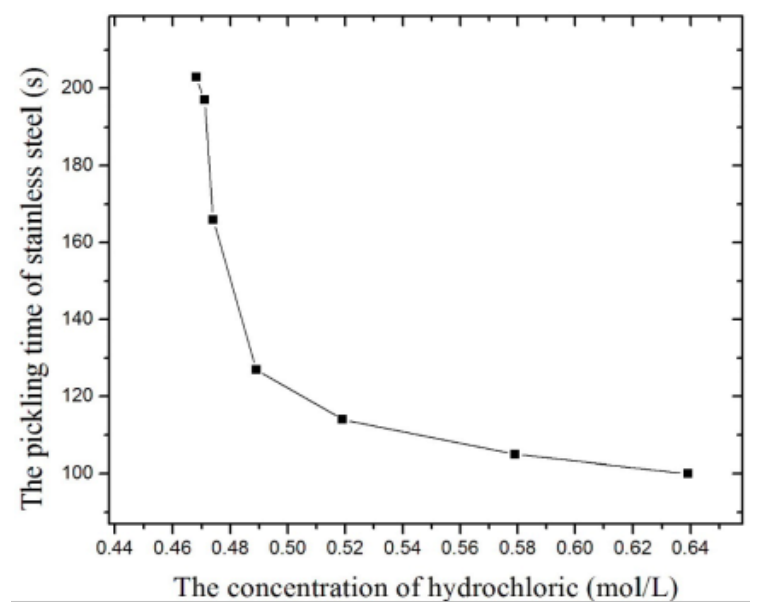

Figure2 The aciding time of different concentration

Firstly, water was pumped to the water tank and the hydrochloric was added to the water. The concentration of the solution was controlled by the ultrasonic liquid level instrument. Secondly, the solution was pumped to the coils of the robot. Thirdly, eight nozzles of the robot completed the two-dimensional positioning through the computer control system. The nozzle moved to the entrance to condenser tubes and the hydrochloric was injected to the condenser tube to immerse the tubes so that the cleaning was finished. When hydrochloric was filled with horizontal condenser tubes, the hydrochloric inside the horizontal tube wouldn't flow out from the horizontal tube even though the cooling water was flowing in vertical pipe because of plunger flow phenomenon. After hydrochloric immersion for a period of time, the nozzle moved to another condenser tube to clean. Then circulating cooling water flowed through the tube and the scale was washed. Eight pipes could be cleaned every time. Then, the nozzles continued to clean the next eight tubes by the movement of the drag chain of the robot.

But there were three problems to be needed to be solved during the process of the robot working. First, how long did it take to immerse; second, whether hydrochloric could cause corrosion for the stainless steel pipe; third, the chloride ion concentration was or not up to the standard.

\section{Experimental}

\subsection{Experimental materials}

In this experiment, the material of the condenser tube was $316 \mathrm{~L}$. The scale was produced on the stainless steel sheet to simulate scale in the inner wall of condenser tubes. Before the experiment, the stainless steel sheet needed to be burnished to facilitate the deposition and adhesion of scale. The stainless steel sheet was cut into and. 


\subsection{Experimental process}

First of all, the corrosion of the samples was tested without the inhibitor. Then, the corrosion rate was calculated under the condition of adding corrosion inhibitor. Finally, the corrosion situation of the sample was observed by electron microscope.

1) Using electronic balance to take quantitative calcium bicarbonate into a beaker, and add deionized water to $60 \mathrm{ml}$. Then to stir it on the magnetic stirrer until thoroughly mixed evenly.

2) Taking a burnished sample to put into acetone solution to clean, then put it under the cold air blower until blown dry. Then weighing the quality and recording.

3) Setting the heater temperature of $30^{\circ} \mathrm{C}$, and to put a sample horizontally into the heater (Figure). Using the dropper to take the quantitative of solution to drop on the plate until the solution evaporated. Repeating the above operation until the solution was completely evaporate. According to the operation, the scale of a certain thickness was made.

4) Putting the sample into the configured acid, and recording the time used in cleaning.

5) Cleaning the pickling plate in the acetone and drying it with the cool air blower. Weighing its quality, then sealing it with a bag.

6) Repeating above experiment, it was concluded that the smallest acid concentration was $0.474 \mathrm{~mol} / \mathrm{L}$.

7) Taking a sample piece () to put it into the solution whose concentration was $0.474 \mathrm{~mol} / \mathrm{L}$ to immerse for 24 hours.

8) Taking out the sample and cleaning it with acetone and then blowing it dry. Then its appearance was observed under the electron microscope.

\section{Results and discussion}

\subsection{Pickling time of different concentration of hydrochloric}

In the experiment, the concentration of hydrochloric was $6 \mathrm{~mol} / \mathrm{L}$. With increasing the volume of hydrochloric, pickling time gradually became shorter. During the experiment, the mass fraction of hydrochloric was 1\%-3\%. The pickling time was 166 seconds when the concentration of hydrochloric was less than $0.474 \mathrm{~mol} / \mathrm{L}$, which was close to the lower limit of online cleaning robot. At this point, the dosage of the corrosion inhibitor was $1.6542 \mathrm{~g}$, whose thickness of scale was 0.5 $\mathrm{mm}$. The corrosion rate conformed to the pickling standard.

With increasing concentration of hydrochloric, corrosion rate showed the tendency of decrease. The reason was that pickling time shortened when the concentration of hydrochloric increased. The conclusion that the corrosion rate of stainless steel sample was less than $1 \mathrm{~g} /\left(\mathrm{m}^{2} \cdot \mathrm{h}\right)$ when the concentration of hydrochloric was of $0.468 \mathrm{~mol} / \mathrm{L}$ to $0.639 \mathrm{~mol} / \mathrm{L}$ could be drew. So, when the mass fraction of hydrochloric was $1.7 \%-3 \%$, the corrosion rate conformed to the standard.

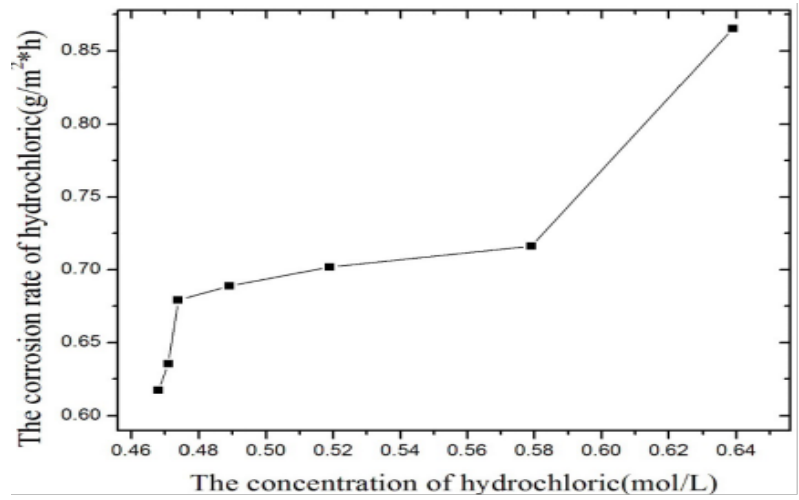

Figure3. The corrosion rate of hydrochloric with different concentration of hydrochloric 


\subsection{The surface corrosion of hydrochloric for stainless steel samples}
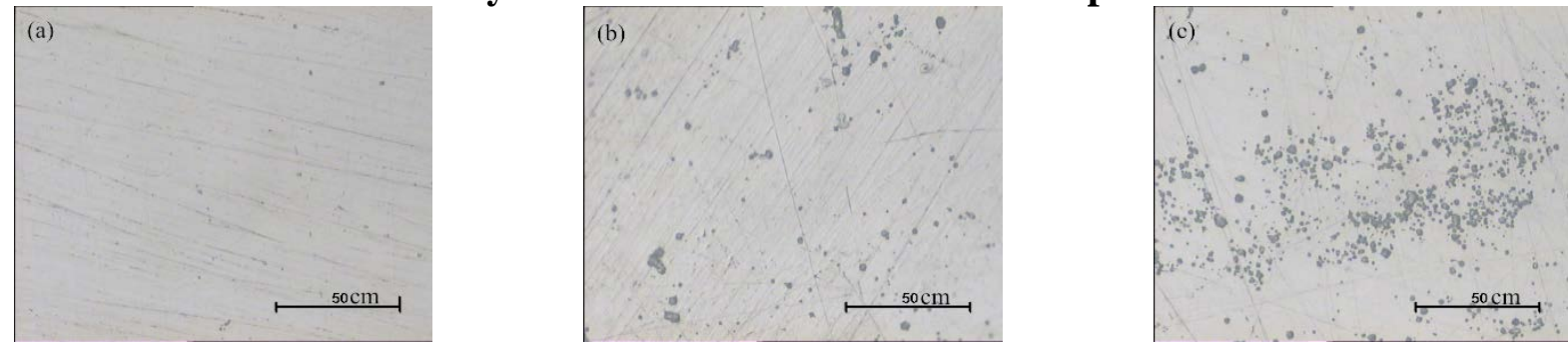

Figure 4. SEM images of the stainless steel surface

In order to study the surface corrosion of hydrochloric for stainless steel samples, the samples was put into the hydrochloric solution and deionized water to immerse for 24 hours.After immersion for 24 hours in hydrochloric solution, there was some slight corrosion on the surface of the stainless steel But there were no pitting corrosion, stress corrosion and so on.

\subsection{The calculation of concentration of chlorine ion in the cooling water system}

According to DL/T 712-712 "power plant condenser and auxiliary cooling pipe material selection", when pickling the stainless steel condenser with hydrochloric, the concentration of chloride ion shall not be higher than 700 ppm.

For a $300 \mathrm{MW}$ unit, the circulation cooling water was $34000 \mathrm{t} / \mathrm{h}$, and the make-up water was $10 \%$ of the circulating water. Under normal circumstances, scale quantity of the condenser tube in the summer was of $71.3-142.6 \mathrm{~kg} / \mathrm{h}$, the winter of $52.8-105.6 \mathrm{~kg} / \mathrm{h}$. When adding hydrochloric to contender tubes whose thickness of the scale was $0.5 \mathrm{~mm}$, the largest quality of hydrochloric was $123.8 \mathrm{~kg}$, whose content of chloride ion was $120.41 \mathrm{~kg}$. The time for cleaning for a $300 \mathrm{MW}$ unit was $124.3 \mathrm{~h}$. The quality of cooling water was $4648820 \mathrm{t}$. The quality of scale whose thickness was $0.5 \mathrm{~mm}$ of 20000 condenser tubes was $25434000 \mathrm{~g}$. The needed quality of hydrochloric was $18566820 \mathrm{~g}$. The content of chlorine ion was $3.88 \mathrm{ppm}$.

Therefore, when hydrochloric was used to remove the scale internal condenser tubes, the concentration of chlorine ion was 3.88 ppm, which was less than 700 ppm.

\section{Summary}

(1) SEM analysis showed that hydrochloric had slight corrosion for condenser tubes during hydrochloric immersion. However, pitting corrosion, stress corrosion and local corrosion did not appear after the whole immersion process of the experiment.

(2) The scale could be cleared within 3 minutes by hydrochloric immersion whose mass fraction was $1.7 \%$ with corrosion inhibitor. This was just in accordance with the working admitted time of online cleaning robot.

(3) The concentration of chloride ion conformed to the prescribed standards.

\section{Reference}

[1] Walker, R., Corrosion Inhabition of Copper by Tolylria-zole, Corrosion, vol. 32, no.8, p. 339-341, 1976.

[2] Cho, Y. I., Lee, S., and Kim, W., Physical Water Treatment for the Mitigation of Mineral Scale in Cooling-Tower Water Applications, in Proceedings of the 2003 ASHRAE Winter Meeting Technical Papers, Chicago, IL, vol. 2, p.113-128, 2003.

[3] Maulbetsch J. Use of degraded water sources as cooling water in power plants.

[4] M. Reza Malayeri and Mohammad Reza Jalalirad, Mitigation of Crystallization Scale in a Single Heated Tube Using Projectiles of Different Sizes and Hardness, Heat Transfer Engineering, vol. 35, pp. 1418-1426, 2014.

[5] Harper W J. Sanitation in dairy food plants. Westport: AVI Pub Company Inc, 1972:167-173. 